Published in final edited form as:

Gastroenterology. 2018 April ; 154(5): 1290-1297.e1. doi:10.1053/j.gastro.2017.12.006.

\title{
No Significant Association Between Proton Pump Inhibitor Use and Risk of Stroke After Adjustment for Lifestyle Factors and Indication
}

\author{
Long H. Nguyen ${ }^{1,2}$, Paul Lochhead ${ }^{1,2}$, Amit D. Joshi ${ }^{1,2}$, Yin Cao ${ }^{1,2,3}$, Wenjie Ma ${ }^{1,2}$, Hamed \\ Khalili $^{1,2}$, Eric B. Rimm ${ }^{3,4,5}$, Kathryn M. Rexrode ${ }^{6}$, and Andrew T. Chan ${ }^{1,2,5,7}$ \\ ${ }^{1}$ Gastrointestinal Unit, Massachusetts General Hospital, Boston, Massachusetts, USA \\ ${ }^{2}$ Clinical and Translational Epidemiology Unit, Massachusetts General Hospital and Harvard \\ Medical School, Boston, Massachusetts, USA \\ ${ }^{3}$ Department of Nutrition, Harvard T.H. Chan School of Public Health, Boston, Massachusetts, \\ USA \\ ${ }^{4}$ Department of Epidemiology, Harvard T.H. Chan School of Public Health, Boston, \\ Massachusetts, USA \\ ${ }^{5}$ Channing Division of Network Medicine, Department of Medicine, Brigham and Women's \\ Hospital, Boston, Massachusetts, USA \\ ${ }^{6}$ Division of Preventive Medicine, Department of Medicine, Brigham and Women's Hospital and \\ Harvard Medical School, Boston, Massachusetts, USA \\ ${ }^{7}$ Broad Institute of Massachusetts Institute of Technology and Harvard, Cambridge, \\ Massachusetts, USA
}

\section{Abstract \\ Background \& Aims-Proton pump inhibitors (PPI) are among the top 10 most-prescribed medications worldwide. We investigated the association between PPI use and ischemic stroke. \\ Methods-We collected data on 68,514 women (mean age 65 57 years) enrolled in the Nurses' Health Study (NHS) since 2000 and 28,989 men (mean age 69 \pm 8 years) in the Health Professionals Follow-up Study since 2004, without a history of prior stroke. We used Cox}

\footnotetext{
Correspondence: Andrew T. Chan, M.D., M.P.H., Professor of Medicine, Clinical and Translational Epidemiology Unit, Massachusetts General Hospital and Harvard Medical School, 55 Fruit Street, GRJ-825C, Boston MA 02114, USA achan@ partners.org.

Disclosures: none

Writing Assistance: none

Author Contributions: study concept and design (LHN, ATC); acquisition of data (LHN); analysis and interpretation of data (LHN, PL, ADJ, YC, WM,HK, EBR, KMR, ATC); drafting of the manuscript (LHN); critical revision of the manuscript (EBR, KMR, ATC); statistical analysis (LHN PL, ADJ); study supervision (ATC)

Publisher's Disclaimer: This is a PDF file of an unedited manuscript that has been accepted for publication. As a service to our customers we are providing this early version of the manuscript. The manuscript will undergo copyediting, typesetting, and review of the resulting proof before it is published in its final citable form. Please note that during the production process errors may be discovered which could affect the content, and all legal disclaimers that apply to the journal pertain.
} 
proportional hazards models to examine the association between risk of incident stroke and PPI use among participants. The primary endpoint was first incident stroke

Results-In the 2 cohorts, we documented 2599 incident strokes (2037 in women and 562 in men) over a 12-year period, encompassing 949,330 person-years. After adjustment for established risk factors for stroke, PPI use was associated with a significant increase in risk of ischemic stroke (hazard ratio, 1.18; 95\% CI, 1.02-1.37). The association was reduced after we adjusted for potential indications for PPI use, including history of peptic ulcer disease, gastroesophageal reflux disease, or gastrointestinal bleeding, and prior use of histamine-2 receptor antagonist therapy (hazard ratio, 1.08; 95\% CI, 0.91-1.27). Regular PPI use was not associated with increased risk of stroke overall or hemorrhagic stroke.

Conclusions-In an analysis of data from the Nurses' Health Study and the Health Professionals Follow-up Study, we did not find a significant association between PPI use and ischemic stroke, after accounting for indications for PPI use. Prior reports of an increased risk of stroke may be due to residual confounding related to chronic conditions associated with PPI use.

\section{Keywords}

epidemiology; reflux treatment; cardiovascular disease; drug

\section{Background \& Aims}

Proton pump inhibitors (PPIs) are a potent class of agents used to suppress gastric acid secretion and are among the most commonly prescribed medications globally ${ }^{1}$. Presently, PPIs are routinely recommended for several gastrointestinal disorders, including gastroesophageal reflux disease (GERD) and prophylaxis against peptic ulcer disease (PUD) and gastrointestinal (GI) bleeding in susceptible populations such as individuals on dual antiplatelet therapy for secondary prevention of cardiovascular disease ${ }^{2,3}$. Several studies have identified a potential association between PPI use and stroke, but have been limited by assessment of PPI use or stroke events through either retrospective recall or administrative claims $^{4-9}$, the inclusion of only individuals with a prior history of cardiovascular disease $(\mathrm{CVD})^{6-12}$, use of composite CVD outcome measures ${ }^{5,7,8,10-12}$, and a lack of detailed information on lifestyle risk factors for stroke or indications for PPI use ${ }^{4-13}$.

Thus, we examined the association between regular PPI therapy and the risk of incident stroke in men and women enrolled in two large cohorts in which detailed information on PPI use as well as other potential health and lifestyle risk factors was collected biennially.

\section{Methods}

\section{Study Population}

The Nurses' Health Study (NHS) is a prospective cohort study of 121,700 female registered nurses aged 30 to 55 years at enrollment in 1976. The Health Professionals Follow-Up Study (HPFS) is a prospective cohort study of 51,529 male health care professionals (dentists, optometrists, pharmacists, podiatrists, and veterinarians) aged 40 to 75 years at enrollment in 1986. Individuals in both cohorts have been followed using detailed biennial questionnaires 
to update information on lifestyle factors, medication use and other exposures of interest, including validated self-assessments of physical activity and a semi-quantitative food frequency questionnaires administered every four years ${ }^{14}, 15$. Follow up rates exceed $90 \%$ of available person-time. This study was approved by the human research committees at the Harvard T. H. Chan School of Public Health and the Brigham and Women's Hospital. Return of study questionnaires was considered to imply informed consent.

\section{Assessment of Incident Stroke}

The primary endpoint was first incident stroke. Upon report of stroke on a biennial questionnaire, we requested permission to review medical records. Physicians blinded to self-reported risk factor status reviewed the retrieved records. Stroke diagnoses were confirmed based on documentation of an episode characterized by a typical neurologic defect attributable to a cerebrovascular event of sudden and rapid onset for $>24$ hours or until death. Stroke was classified as ischemic (thrombotic, embolic, or non-hemorrhagic), hemorrhagic (intraparenchymal hemorrhage or subarachnoid hemorrhage), or unknown type, consistent with well-established criteria ${ }^{16}$. If medical records were not available, the case was considered probable. We included confirmed and probable cases for analysis. Deaths were confirmed by reporting from next of kin, co-workers, or postal authorities or by searching the National Death Index.

\section{Assessment of PPI Usage}

Every two years since inception, participants have been asked to report medications they had used regularly in the preceding two years including histamine-2 receptor antagonists (H2RAs) starting in 1980. Beginning in the year 2000 for the NHS and 2004 for the HPFS and for every subsequent two-year period thereafter, participants were specifically asked whether they used PPIs regularly. While examples of brand names were provided for reference, specific information on brand, dose, and schedule was not specifically queried.

\section{Assessment of Additional Covariates}

Information on potential confounding factors including age (continuous), smoking status (pack-years, continuous), menopausal hormone therapy in women (current vs. never/past), body mass index (BMI, continuous), regular use of multivitamins (yes/no), aspirin, or nonsteroidal anti-inflammatory drug usage ( $\geq 2$ tablets per week; yes/no for each), personal history of hypertension, hyperlipidemia, diabetes mellitus, or coronary heart disease (yes/no for each) and other medical diagnoses and lifestyle behaviors were updated biennially, with diet quality (Alternative Healthy Eating Index), alcohol intake (g/day, continuous), and physical activity (quintiles of metabolic equivalent task [MET]-hours/week) updated every four years. Further, information on several common indications for PPI usage including history of peptic ulcer disease, gastroesophageal reflux disease, or gastrointestinal bleeding (each yes/no) were asked regularly. The validity of dietary assessment, anthropomorphic measurements, and physical activity has been previously reported ${ }^{14,17-22}$. 


\section{Statistical Analysis}

We began follow-up with the date of return of the 2000 questionnaire in the NHS and the 2004 questionnaire in the HPFS, the questionnaire cycles in which PPI use was first queried. We excluded participants with a personal history of prior stroke or cancer before baseline and those with missing data on our exposure of interest. After these exclusions, the final study population consisted of 68,514 women and 28,989 men. Person-time for each participant was accrued from baseline to the date of first confirmed stroke, death from any cause, date of last returned questionnaire, or June 1, 2012 in NHS and January 1, 2012 in HPFS, whichever occurred first. Individuals were defined as PPI users if they responded that they regularly used PPI in the preceding two-years. We employed Cox proportional hazards modeling using time-varying variables with the most updated information for PPI use and other covariates of interest before each two-year time interval to calculate adjusted hazard ratios and their 95\% confidence intervals. We also examined PPI use and stroke risk according to strata of other risk factors. We also performed several sensitivity analyses to support our primary findings. The $Q$-test was used to test for heterogeneity in the association of PPI use and stroke between study cohorts and random effects modeling was employed to produce summary estimates. SAS version 9.4 (Cary, NC, USA) was used for all statistical analysis. All $p$-values were two-tailed with values $₫ \mathbf{} 0.05$ considered statistically significant.

\section{Results \\ Cohort Characteristics}

At baseline, among the 97,503 participants (68,514 women and 28,989 men), mean age $69 \pm 8$ years, $6.5 \%$ of women and $16.1 \%$ of men reported regular PPI use. The rate of PPI use at that time was comparable to the rate of other U.S. populations ${ }^{23-25}$. Participants who were regular PPI users at baseline tended to have higher rates of chronic medical conditions including prior history of hypertension, hyperlipidemia, coronary artery disease, and diabetes mellitus, as well as lower levels of physical activity expenditure (Table 1). Among women, regular PPI users were more likely to have a higher BMI and a prior history of menopausal hormone use and higher BMI. As expected, PPI users had considerably higher rates of prior peptic ulcer disease (PUD), GERD, prior gastrointestinal (GI) bleeding, or prior use of H2RAs.

\section{PPI Use and Incident Stroke}

Over 12 years encompassing 949,330 person-years of follow-up, we documented 2,599 incident strokes (2,037 in women and 562 in men). In age-adjusted models comparing PPI users to non-users, there was no observed difference in the combined cohort in the summary estimate of risk for total stroke (HR 1.11, 95\% CI: 1.00-1.24) or hemorrhagic stroke (HR 1.00, 95\% CI: 0.72-1.38; Table 2). We did observe a statistically significant difference in age-adjusted risk for ischemic stroke (HR 1.25, 95\% CI: 1.08-1.46). This association was attenuated somewhat, but remained statistically significant after multivariable adjustment for smoking status, BMI, physical activity, dietary quality, alcohol use, menopausal hormone use in women, multivitamin use, regular aspirin use, regular non-aspirin NSAID use, and personal history of hypertension, hyperlipidemia, coronary artery disease, or diabetes (HR $1.18,95 \%$ CI: $1.02-1.37)$. 
To address the possibility that clinical indications for PPI use may confound the association of PPI use with ischemic stroke, we conducted additional analyses adjusting for several indications for PPI use including history of PUD, GERD, prior GI bleeding, or prior use of H2RAs. With this additional adjustment, the association between PPI use and ischemic stroke was further attenuated (HR 1.08, 95\% CI: 0.91-1.27). Of note, there was no single indication that was the dominant source of confounding.

Given their shared indications of use, we examined the influence of H2RA use on risk for stroke. In age-adjusted analyses, regular users of H2RAs were at modestly increased risk of total stroke, though this was attenuated in multivariate testing. There was no apparent association between H2RA use and ischemic stroke (Supplemental Table 1). There was no clear association between duration of PPI use and risk of ischemic stroke $\left(\mathrm{P}_{\text {trend }}=0.18\right.$; Table $3)$.

We evaluated potential differences in the association between regular PPI use and stroke according to strata of known stroke risk factors. The estimates for risk of ischemic stroke among PPI users did not appear to differ according to several stroke risk factors, including subgroups defined by age, regular aspirin use, hypertension, or hyperlipidemia. However, the association of PPI use with ischemic stroke appeared to be of greater magnitude among participants with obesity, or a prior history of diabetes or coronary heart disease, though formal tests for interaction were not statistically significant (Table 4; all $\mathrm{P}_{\text {interaction }} \searrow^{0.10}$ ).

To ensure that PPI use was not predictive of later stroke development, we performed a twoyear lag analysis in which exposure information was derived two questionnaire cycles (approximately four years) prior to the follow-up interval. The association of PPI use with ischemic stroke was not materially altered (multivariable HR 0.93, 95\% CI: 0.77-1.12).

\section{Conclusions}

In this prospective investigation, we observed an association between regular PPI use and risk of ischemic stroke in age-adjusted models and multivariate models adjusting for known risk factors for ischemic stroke that was consistent with the findings of prior reports. However, additional adjustment for factors that may be associated with initiation and continuation of PPI therapy, including history of peptic ulcer disease, GERD, GI bleeding, and prior use of H2RA therapy substantially attenuated the association. Taken together, these data suggest that the association of PPI use with ischemic stroke may be due to residual confounding by factors associated with the indication for PPI use.

Our finding of a modestly increased risk for ischemic stroke associated with regular PPI use that was subsequently attenuated after further adjustment for several indications for PPI use may be explained by confounding from factors incompletely accounted for in our multivariable model. For example, the metabolic syndrome is a shared risk factor between GERD and ischemic stroke. Thus, adjusting for GERD may better control for confounding associated with the metabolic syndrome which is not completely accounted for with adjustment for cardiac risk factors. 
The risk of ischemic stroke and PPI therapy has been previously studied most often in the context of populations with established CVD at baseline or as part of a composite outcome inclusive of other vascular events including myocardial infarction. Our findings contrast with two recent publications that specifically focused on PPI use and ischemic stroke, including results from a retrospective case-control study of the Taiwan National Insurance Database which observed a modestly increased risk for first-time ischemic stroke within a 120-day period of initiating PPI use ${ }^{4}$. In their propensity-based analysis, Wang et al found that PPI use was associated with an age-adjusted increased risk for ischemic stroke (HR 1.36, 95\% CI: 1.14-1.62) which appeared to be of somewhat stronger magnitude than the age-adjusted risk that we observed (HR 1.25, 95\% CI: 1.08-1.46). This may be due to the shorter followup period (120 days) compared to our study. With shorter follow-up, the likelihood of confounding by clinical indications for PPI use may be more evident. For example, more acute health issues associated with near-term PPI use may also be associated with stroke. In fact, in their nested case-control analysis which extended the follow up period, the observed risk for ischemic stroke actually weakened with increasing duration of therapy. Finally, although propensity score matching was employed in the analyses, factors contributing to the score were limited to chronic conditions such as diabetes, chronic kidney disease, drug abuse, physical limitation, autoimmune disease, and prior history of cancer. Therefore, confounding by factors associated with PPI use or the likelihood of receiving acidsuppression therapy may still have occurred.

In a separate investigation leveraging Danish national registry data, Sehested et al found that PPI use determined by pharmacy records was associated with ischemic stroke (HR 1.13, 95\% CI: 1.09-1.19) based on billing data after adjustment for several indications for therapy including PUD, GI hemorrhage, and GERD ${ }^{5}$. Further, they noted this association was more apparent with high-dose PPI (HR 1.31, 95\% CI: 1.21-1.42). Our results may have differed because our PPI exposure was based on self-report, which more likely captured actual intake, and our stroke endpoints were based on adjudicated medical record review, which is less prone to misclassification. Furthermore, in our analysis, we also adjusted for H2RA usage which may further account for unmeasured confounding. Although we acknowledge that we lacked dosage information which precludes direct comparison with the Danish study, their study notably used a limited definition of dose based only within a six-month inclusion period. Additionally, study inclusion criteria required a history of elective gastroscopy, raising the possibility of selection bias as well as lead-in bias as subjects were likely exposed to PPI therapy prior to study baseline, limiting generalizability. For example, over $40 \%$ of individuals in this study filled a PPI prescription without accounting for over-the-counter usage during the six-year follow-up period, a relatively high number compared to the general population.

Our study includes several strengths. First, compared to prior analyses, we had prospective, biennially updated collection of both PPI use and several important potential stroke risk factors. Second, our long-term follow-up and rigorous adjudication of stroke events is a significant advantage over prior case-control studies of large claims databases which rely on discharge coding, which may be inaccurate. Third, in this group of health professionals, ascertainment of PPI use by self-reporting is likely to be a more accurate reflection of actual use, including prescription and over-the-counter sources, compared with studies based on 
prescription data alone. Finally, we had the ability to adjust for other potential indications for PPI use.

We acknowledge several limitations of this study. PPI users differed from nonusers in many attributes including a higher burden of chronic medical conditions. As with any observational study design, there remains the possibility of additional residual and unmeasured confounding. However, we did adjust for many more available potential confounding variables in our analysis than comparable studies which substantially attenuated modestly significant associations. Finally, we did not collect information on PPI brand, dosage, and schedule though most studies have suggested any potential association is a class effect not associated with any one specific drug. However, Sehested et al did observe a stronger association with high-dose PPI therapy, which we were unable to directly assess.

In this prospective study of two large, population-based cohorts, regular PPI therapy was not associated with an increase in the risk of incident ischemic stroke after adjustment for multiple stroke risk factors and potential indications for PPI use. These findings suggest that previously reported associations relating PPI therapy and ischemic stroke or other adverse events may have resulted from residual confounding, highlighting the need for caution in the interpretation of pharmacoepidemiologic data in which modest associations are detected.

\section{Supplementary Material}

Refer to Web version on PubMed Central for supplementary material.

\section{Acknowledgments}

Grant Support: NIH UM1 CA186107 (NHS), NIH UM1 CA167552 (HPFS), NIH R01 HL35464 (EBR), NHLBI R01 HL088521(KMR), and NIH K24 DK098311 (ATC).

We are indebted to the participants in the Nurses' Health Study (NHS) and the Health Professionals Follow-up Study (HPFS) for their participation. This research was supported by the following grants: NIH UM1 CA186107 (NHS), NIH UM1 CA167552 (HPFS), NIH R01 HL35464 (EBR), NHLBI R01 HL088521(KMR), and NIH K24 DK098311 (ATC).

\section{Abbreviations}

$\begin{array}{ll}\text { BMI } & \text { body mass index } \\ \text { CVD } & \text { cardiovascular disease } \\ \text { GERD } & \text { gastroesophageal reflux disease } \\ \text { GI } & \text { gastrointestinal } \\ \text { H2RA } & \text { histamine-2 receptor antagonist } \\ \text { HPFS } & \text { Health Professionals Follow Up Study } \\ \text { NHS } & \text { Nurses' Health Study } \\ \text { PPI } & \text { proton pump inhibitor }\end{array}$


PUD peptic ulcer disease

\section{References}

1. Kantor ED, Rehm CD, Haas JS, et al. Trends in Prescription Drug Use Among Adults in the United States From 1999-2012. JAMA. 2015; 314:1818-31. [PubMed: 26529160]

2. Levine GN, Bates ER, Bittl JA, et al. 2016 ACC/AHA Guideline Focused Update on Duration of Dual Antiplatelet Therapy in Patients With Coronary Artery Disease: A Report of the American College of Cardiology/American Heart Association Task Force on Clinical Practice Guidelines: An Update of the 2011 ACCF/AHA/SCAI Guideline for Percutaneous Coronary Intervention, 2011 ACCF/AHA Guideline for Coronary Artery Bypass Graft Surgery, 2012 ACC/AHA/ACP/AATS/ PCNA/SCAI/STS Guideline for the Diagnosis and Management of Patients With Stable Ischemic Heart Disease, 2013 ACCF/AHA Guideline for the Management of ST-Elevation Myocardial Infarction, 2014 AHA/ACC Guideline for the Management of Patients With Non-ST-Elevation Acute Coronary Syndromes, and 2014 ACC/AHA Guideline on Perioperative Cardiovascular Evaluation and Management of Patients Undergoing Noncardiac Surgery. Circulation. 2016; 134:e123-55. [PubMed: 27026020]

3. Li L, Geraghty OC, Mehta Z, et al. Age-specific risks, severity, time course, and outcome of bleeding on long-term antiplatelet treatment after vascular events: a population-based cohort study. Lancet. 2017

4. Wang YF, Chen YT, Luo JC, et al. Proton-Pump Inhibitor Use and the Risk of First-Time Ischemic Stroke in the General Population: A Nationwide Population-Based Study. Am J Gastroenterol. 2017

5. Sehested TSG, Gerds TA, Fosbol EL, et al. Long-term use of Proton Pump Inhibitors, Doseresponse Relationship, and Associated Risk of Ischemic Stroke and Myocardial Infarction. J Intern Med. 2017

6. Leonard CE, Bilker WB, Brensinger CM, et al. Comparative risk of ischemic stroke among users of clopidogrel together with individual proton pump inhibitors. Stroke. 2015; 46:722-31. [PubMed: 25657176]

7. Charlot M, Grove EL, Hansen PR, et al. Proton pump inhibitor use and risk of adverse cardiovascular events in aspirin treated patients with first time myocardial infarction: nationwide propensity score matched study. BMJ. 2011; 342:d2690. [PubMed: 21562004]

8. Stockl KM, Le L, Zakharyan A, et al. Risk of rehospitalization for patients using clopidogrel with a proton pump inhibitor. Arch Intern Med. 2010; 170:704-10. [PubMed: 20421557]

9. Juurlink DN, Gomes T, Mamdani MM, et al. The safety of proton pump inhibitors and clopidogrel in patients after stroke. Stroke. 2011; 42:128-32. [PubMed: 21164123]

10. Goodman SG, Clare R, Pieper KS, et al. Association of proton pump inhibitor use on cardiovascular outcomes with clopidogrel and ticagrelor: insights from the platelet inhibition and patient outcomes trial. Circulation. 2012; 125:978-86. [PubMed: 22261200]

11. Nicolau JC, Bhatt DL, Roe MT, et al. Concomitant proton-pump inhibitor use, platelet activity, and clinical outcomes in patients with acute coronary syndromes treated with prasugrel versus clopidogrel and managed without revascularization: insights from the Targeted Platelet Inhibition to Clarify the Optimal Strategy to Medically Manage Acute Coronary Syndromes trial. Am Heart J. 2015; 170:683-694. e3. [PubMed: 26386792]

12. Yi X, Zhou Q, Wang C, et al. Concomitant Use of Proton Pump Inhibitors and Clopidogrel Is Not Associated with Adverse Outcomes after Ischemic Stroke in Chinese Population. J Stroke Cerebrovasc Dis. 2016; 25:2859-2867. [PubMed: 27546731]

13. Charlot M, Ahlehoff O, Norgaard ML, et al. Proton-pump inhibitors are associated with increased cardiovascular risk independent of clopidogrel use: a nationwide cohort study. Ann Intern Med. 2010; 153:378-86. [PubMed: 20855802]

14. Wolf AM, Hunter DJ, Colditz GA, et al. Reproducibility and validity of a self-administered physical activity questionnaire. Int J Epidemiol. 1994; 23:991-9. [PubMed: 7860180]

15. Rimm E, Giovannucci E, Stampfer M, et al. Reproducibility and validity of an expanded selfadministered semiquantitative food questionnaire among health professionals. Am J Epidemiol. 1992; 135:1114-1126. [PubMed: 1632423] 
16. Walker AE, Robins M, Weinfeld FD. The National Survey of Stroke. Clinical findings Stroke. 1981; 12:I13-44. [PubMed: 7222164]

17. Troy LM, Hunter DJ, Manson JE, et al. The validity of recalled weight among younger women. Int J Obes Relat Metab Disord. 1995; 19:570-2. [PubMed: 7489028]

18. Khalili H, Huang ES, Jacobson BC, et al. Use of proton pump inhibitors and risk of hip fracture in relation to dietary and lifestyle factors: a prospective cohort study. BMJ. 2012; 344:e372. [PubMed: 22294756]

19. Lin BM, Curhan SG, Wang M, et al. Prospective Study of Gastroesophageal Reflux, Use of Proton Pump Inhibitors and H2-Receptor Antagonists, and Risk of Hearing Loss. Ear Hear. 2017; 38:2127. [PubMed: 27556519]

20. Iso H, Rexrode KM, Stampfer MJ, et al. Intake of fish and omega-3 fatty acids and risk of stroke in women. JAMA. 2001; 285:304-12. [PubMed: 11176840]

21. Chiuve SE, Rexrode KM, Spiegelman D, et al. Primary prevention of stroke by healthy lifestyle. Circulation. 2008; 118:947-54. [PubMed: 18697819]

22. Colditz GA, Martin P, Stampfer MJ, et al. Validation of questionnaire information on risk factors and disease outcomes in a prospective cohort study of women. Am J Epidemiol. 1986; 123:894900. [PubMed: 3962971]

23. Jacobson BC, Ferris TG, Shea TL, et al. Who is Using Chronic Acid Suppression Therapy and Why[quest]. Am J Gastroenterol. 2003; 98:51-58. [PubMed: 12526936]

24. Rane P, Aparasu RR, Guha S. Proton Pump inhibitor prescribing trend in the us Ambulatory setting. Value in Health. 18:A265.

25. Targownik LE, Metge C, Roos L, et al. The Prevalence of and the Clinical and Demographic Characteristics Associated With High-Intensity Proton Pump Inhibitor Use. Am J Gastroenterol. 2007; 102:942-950. [PubMed: 17313495] 
TABLE 1

AGE-STANDARDIZED BASELINE CHARACTERISTICS ACCORDING TO PPI USE

Nurses' Health Study (2000)

Non-user $(n=64,055)$

\begin{tabular}{lc}
\hline Age, years & 65.7 (7.1) \\
Smoking, \% & \\
$\quad$ Never & 45 \\
$\quad$ Past & 46 \\
Current & 9 \\
Exercise, mean MET-hrs/wk & $17.5(22.1)$ \\
BMI, kg $2 / \mathrm{mg}^{2}$ & $26.6(5.3)$ \\
Regular ASA usage, \% & 26 \\
Regular NSAID usage, \% & 28 \\
Past/current PMH, \% & 46 \\
AHEI score & $50.0(9.6)$ \\
Hypertension, \% & 47 \\
Hyperlipidemia, \% & 59 \\
Coronary Heart Disease, $\%$ & 3 \\
Diabetes Mellitus, \% & 8 \\
GERD, \% & 8 \\
Prior PUD, \% & 9 \\
Prior GI bleed, \% & 2 \\
Past/current H2RA usage, \% & 15 \\
\hline
\end{tabular}

42

7

24

29

52

63

73

5

11

37

28

6

66
Health Professionals Follow-Up Study (2004)

Regular user $(n=4,459)$

$65.7(7.1)$

Non-user $(n=24,326)$

$69.9(8.6)$

Regular user ( $n=4663$ )

$69.9(8.5)$

53

49

$13.9(18.2)$

$28.3(5.8)$

$49.4(9.4)$
44

48

3

45.4 (47.3)

40.4 (45.2)

$25.6(4.9)$

59

25.9 (4.9)

18

N/A

$52.4(10.8)$

49

59

62

20

N/A

$51.8(10.5)$

60

68

12

9

10

11

23

72

$9 \quad 22$

$4 \quad 8$

12

49

Values are means (SD) or percentages and are standardized to the age distribution of the study population.

MET: metabolic-equivalent task, BMI: body mass index, ASA: acetylsalicylic acid, NSAID: non-steroidal anti-inflammatory drug, AHEI: Alternative Healthy Eating Index, GERD: gastroesophageal reflux disease, PUD: peptic ulcer disease, GI: gastrointestinal, H2RA: histamine-2 receptor antagonist

Regular use of either ASA or NSAIDs defined as $\geq 2$ tablets per week. PMH use measured only among post-menopausal females. 
TABLE 2

RISK OF STROKE EVENTS BY CURRENT USE OF PPI THERAPY

\begin{tabular}{llcc}
\hline \multicolumn{1}{l}{ TOTAL STROKE } & & \\
\hline NHS & & NON-USER & REGULAR USER \\
& Cases/Person-years & $1,707 / 635,985$ & $330 / 110,756$ \\
& Age-adjusted & 1 (Ref.) & $1.14(1.01-1.29)$ \\
& Model 1 & 1 (Ref.) & $1.07(0.95-1.21)$ \\
& Model 2 & 1 (Ref.) & $1.01(0.89-1.16)$ \\
\hline HPFS & Cases/Person-years & $467 / 170,856$ & $95 / 31,733$ \\
& Age-adjusted & 1 (Ref.) & $1.03(0.82-1.29)$ \\
& Model 1 & 1 (Ref.) & $1.00(0.80-1.25)$ \\
& Model 2 & 1 (Ref.) & $0.89(0.69-1.14)$ \\
\hline \multirow{2}{*}{ TOTAL } & Cases/Person-years & $2,174 / 806,841$ & $425 / 142,489$ \\
& Age-adjusted & 1 (Ref.) & $1.11(1.00-1.24)$ \\
& Model 1 & 1 (Ref.) & $1.06(0.95-1.17)$ \\
& Model 2 & 1 (Ref.) & $0.99(0.88-1.11)$
\end{tabular}

ISCHEMIC STROKE

NON-USER REGULAR USER

\begin{tabular}{llcc}
\hline NHS & Cases/Person-years & $811 / 635,985$ & $169 / 110,756$ \\
& Age-adjusted & 1 (Ref.) & $1.27(1.07-1.51)$ \\
& Model 1 & 1 (Ref.) & $1.18(0.99-1.40)$ \\
& Model 2 & 1 (Ref.) & $1.08(0.89-1.30)$ \\
\hline HPFS & Cases/Person-years & $225 / 170,856$ & $56 / 31,733$ \\
& Age-adjusted & 1 (Ref.) & $1.21(0.90-1.62)$ \\
& Model 1 & 1 (Ref.) & $1.18(0.88-1.60)$ \\
& Model 2 & 1 (Ref.) & $1.08(0.77-1.51)$ \\
\hline \multirow{2}{*}{ TOTAL } & Cases/Person-years & $1,036 / 806,841$ & $225 / 142,489$ \\
& Age-adjusted & 1 (Ref.) & $1.25(1.08-1.46)$ \\
& Model 1 & 1 (Ref.) & $1.18(1.02-1.37)$ \\
& Model 2 & 1 (Ref.) & $1.08(0.91-1.27)$
\end{tabular}

HEMORRHAGIC STROKE

\begin{tabular}{llcc} 
& & NON-USER & REGULAR USER \\
\hline \multirow{2}{*}{ NHS } & Cases/Person-years & $223 / 635,985$ & $36 / 110,756$ \\
& Age-adjusted & 1 (Ref.) & $0.94(0.65-1.35)$ \\
& Model 1 & 1 (Ref.) & $0.97(0.67-1.40)$ \\
& Model 2 & 1 (Ref.) & $0.94(0.63-1.39)$ \\
\hline \multirow{2}{*}{ HPFS } & Cases/Person-years & $50 / 170,856$ & $12 / 31,733$ \\
& Age-adjusted & 1 (Ref.) & $1.19(0.63-2.25)$
\end{tabular}




\begin{tabular}{llcc} 
& Model 1 & 1 (Ref.) & $1.12(0.59-2.15)$ \\
& Model 2 & 1 (Ref.) & $0.79(0.38-1.63)$ \\
\hline TOTAL & Cases/Person-years & $273 / 806,841$ & $48 / 142,489$ \\
& Age-adjusted & 1 (Ref.) & $0.99(0.72-1.36)$ \\
& Model 1 & 1 (Ref.) & $1.01(0.73-1.38)$ \\
& Model 2 & 1 (Ref.) & $0.90(0.64-1.27)$ \\
\hline
\end{tabular}

Model 1: adjusted for age (continuous), smoking status (never $<5,5-20,20-40,>40$ pack-years), alcohol intake (g/day, continuous), body-mass index (continuous), physical activity (metabolic-equivalent task/week, continuous), Alternative Healthy Eating Index scores (continuous), menopausal hormone use (among women, current vs. past/never), multivitamin use, regular aspirin use, regular non-aspirin NSAID use, history of hypertension, hyperlipidemia, coronary artery disease, or diabetes

Model 2: Model 1 + history of peptic ulcer disease (ever/never), history of gastroesophageal reflux disease (GERD; ever/never), history of gastrointestinal bleeding (ever/never), history of prior histamine-2 receptor antagonist (H2RA) usage (ever/never) 
Nguyen et al.

Page 14

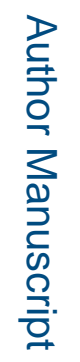

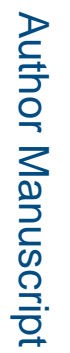

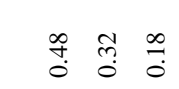

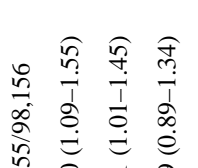

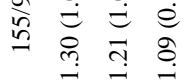

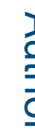

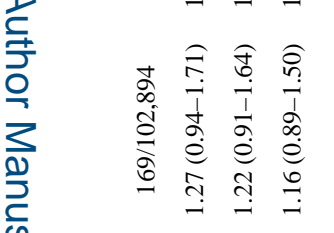

言

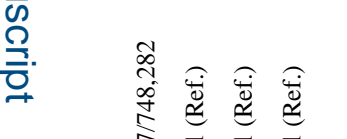

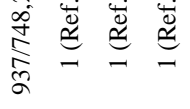

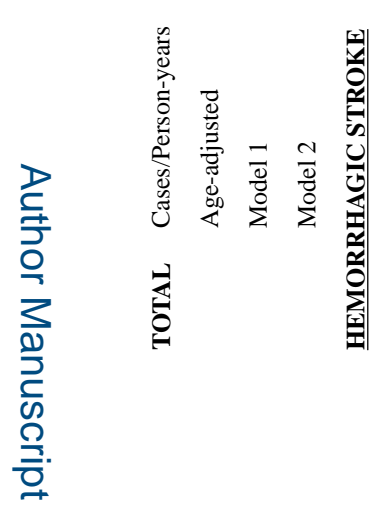

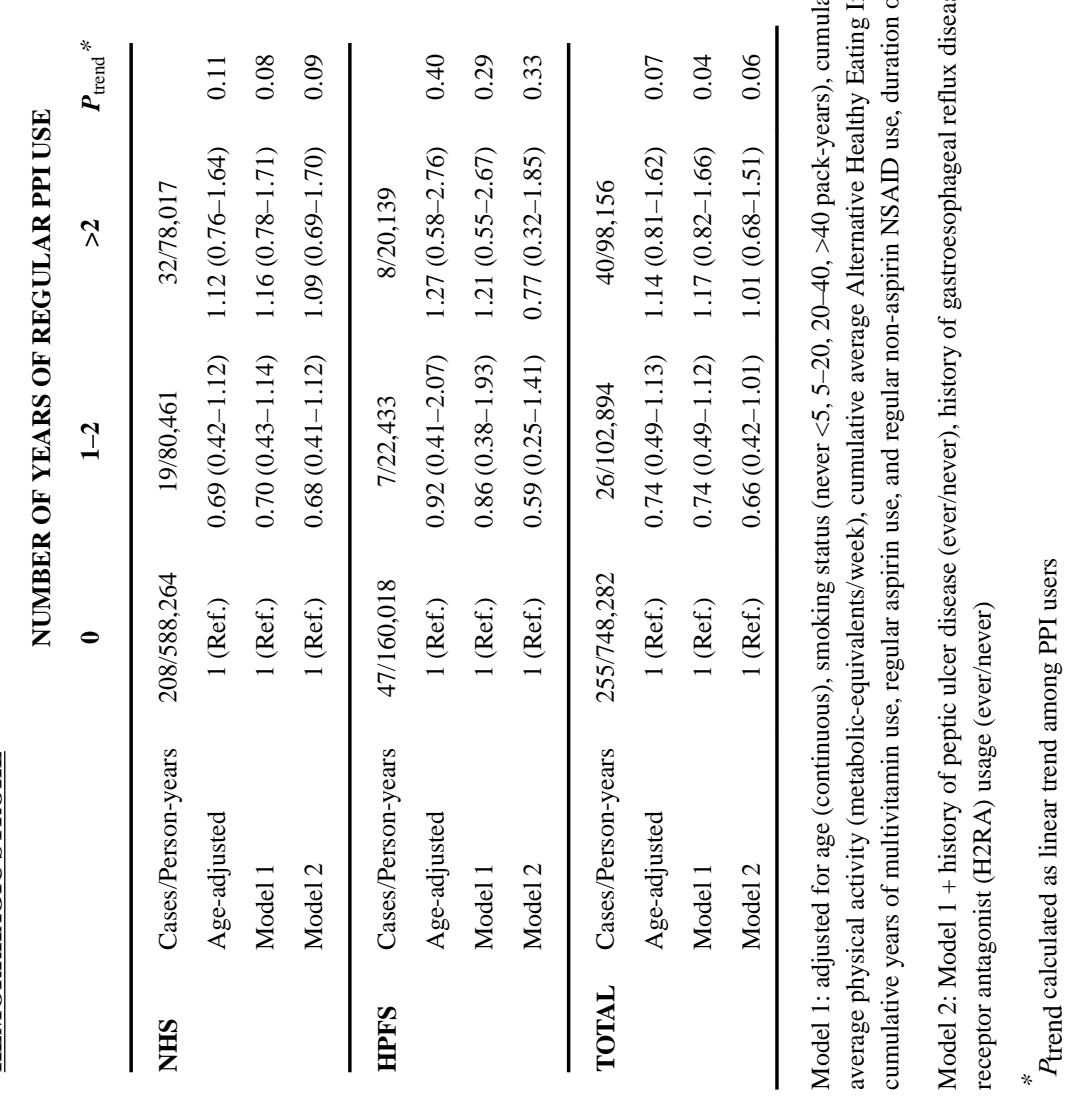

Gastroenterology. Author manuscript; available in PMC 2019 April 01. 


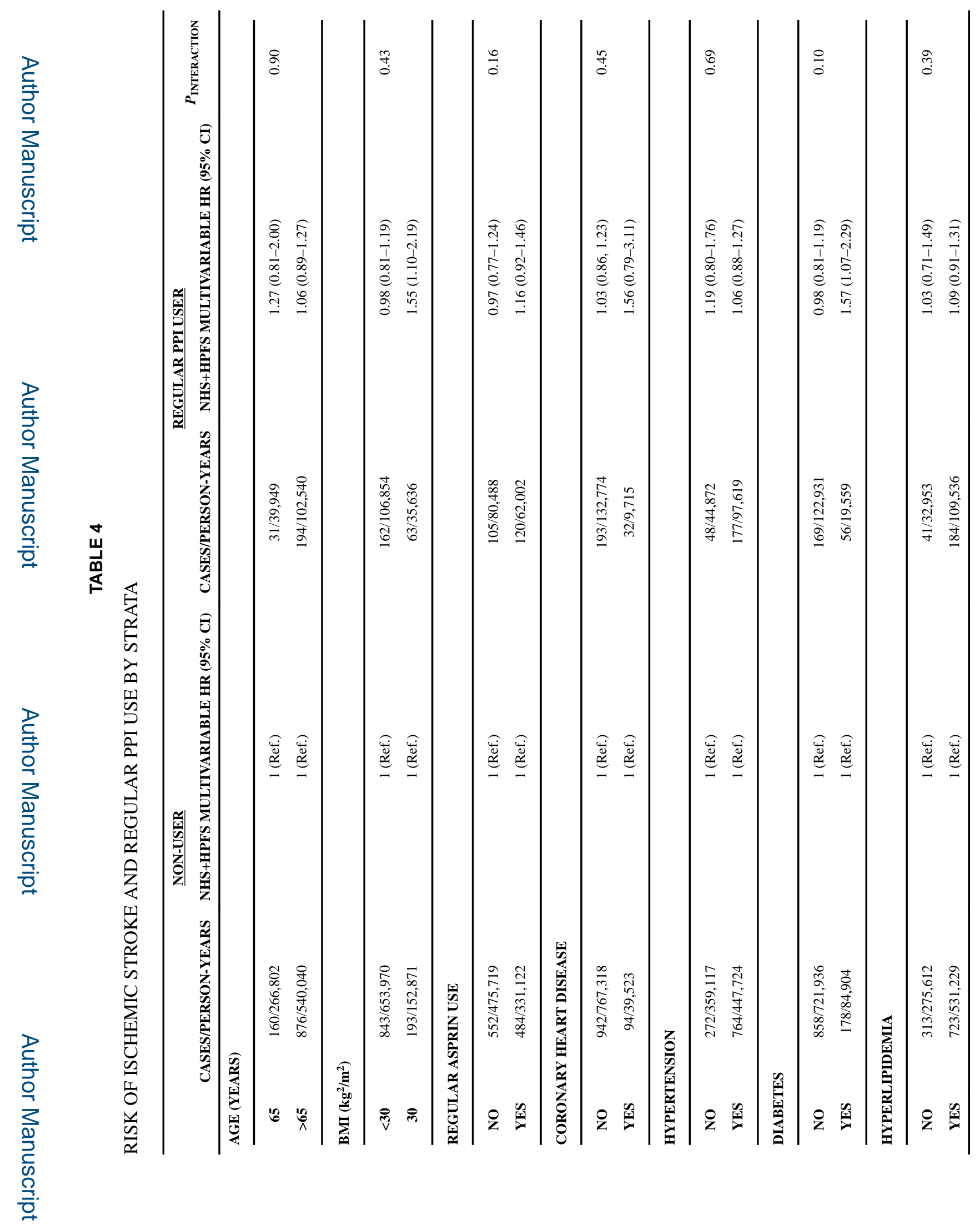

Gastroenterology. Author manuscript; available in PMC 2019 April 01. 


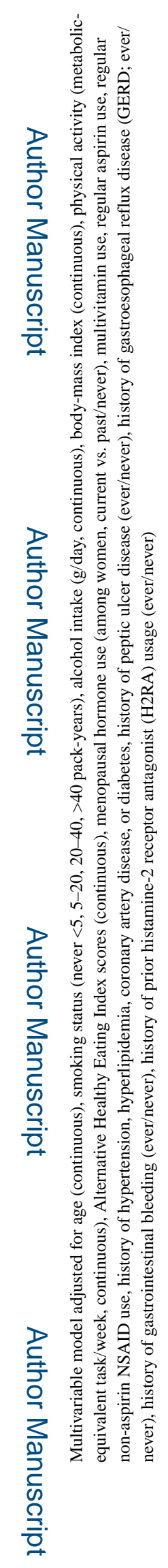

Gastroenterology. Author manuscript; available in PMC 2019 April 01. 